\section{Neutrons and the Origin of Life}

WHEN in early days of the development of the earth its surface was covered with an aseptic solution of various organic compounds, these did not react in the same way as they would have done if certain enzymes, colloids and other complicated substances had already been present ${ }^{1}$. The very first impetus to further development had to be given from molecules, containing, for example, carbon and nitrogen in certain combinations. One possible way of combining nitrogen and carbon, not yet discussed in this connexion, may be found in conjunction with certain nuclear reactions, also prevalent in Nature at those early days.

By the action of cosmic rays or hard $\gamma$-rays from radioactive matter present in the ocean, the deuterium of the heavy water, contained in the ocean water, will produce neutrons. These neutrons are slowed down in the water and will react with the nuclei of atoms in the molecules dissolved in the water. Thus, for example, a heavy carbon atom in an already existent carbon chain compound should be transformable (through a short-lived radioactive carbon isotope) into a stable nitrogen atom by expulsion of a $\beta$-particle :

$$
{ }_{6}^{13} \mathrm{C}+{ }_{0}^{1} n \rightarrow{ }_{6}^{14} \mathrm{C}^{*} \rightarrow{ }_{7}^{14} \mathrm{~N}+-1 \beta
$$

If the molecule in which this reaction takes place is not destroyed by the recoil or by the $\beta$-particle, the new nitrogen atom may give rise to a new, rather unexpected molecule containing a carbonnitrogen link and capable of new reactions. In the same way also a phosphorus atom may be generated from a silicon atom and so on.

Even the direct action of very hard $\gamma$-rays on oxygen $^{2}$ is capable of producing a stable nitrogen atom (with mass number 15) through a short-lived radioactive oxygen isotope :

$$
\begin{aligned}
{ }_{8}^{16} \mathrm{O}+\gamma & \rightarrow{ }_{8}^{15} \mathrm{O}^{*}+{ }_{0}^{1} n \\
{ }_{8} \mathrm{O}^{*} & \rightarrow{ }^{15} \mathrm{~N}+{ }_{1}^{0} e
\end{aligned}
$$

Thus various complicated organic molecules containing, for example, carbon-nitrogen may have originated in a way rather different from ordinary chemical reactions and will perhaps have influenced the evolution of organic matter, eventually leading to the first primitive forms of living matter, no longer present on earth.

\section{Electrolux Laboratory, Stockholm.}

Aug. 15.

'Compare Oparin, A. J., "The Origin of Life" (New York, 1938). 'Chang, Goldhaber and Sagane, NATURE, 139, 962 (1937).

\section{Condensation Coefficients of Mercury Halides}

Using the method of Alty ${ }^{1}$, we have measured the coefficients of condensation $(f)$ of the compounds mercuric chloride, bromide and iodide. A hemispherical cap of these salts was deposited by sub. limation on the end of a glass tube which could be heated internally by boiling turpentine. The temperature of the cap surface was measured by a fine thermocouple while the salt evaporated in a high vacuum. The quantity of salt evaporated per unit time and area $\left(M_{1}\right)$ was determined by weighing the deposit on a surrounding glass tube immersed in liquid air. By calculating the maximum rate of evaporation $\left(M_{2}\right)$ from the formula of the kinetic theory of gases,

$$
M_{2}=\frac{0.0584 \sqrt{ } M}{t} \int^{t} \frac{P}{\sqrt{ } T} d t \mathrm{gm} . / \mathrm{cm} .{ }^{2} \text { sec. }
$$

where $P$ is saturated vapour pressure ( $\mathrm{mm}$. mercury), $M$ is molecular weight, $t$ is time of evaporation, $T$ is absolute temperature, one finds $f=M_{1} / M_{2}$.

The mean of the results of three or four runs for each substance is as follows :

$$
\begin{array}{ccc}
\mathrm{HgCl}_{2} & \mathrm{HgBr}_{2} & \mathrm{HgI}_{3} \\
0.76 \pm 0.1 & 0.64 \pm 0.1 & 0.53 \pm 0.1
\end{array}
$$

For calculating $M_{2}$ the saturated vapour pressures $P$ were extrapolated from the formulæ given in the "Critical Tables". The extrapolated values seem to be a little too small as compared with single direct measurements ${ }^{2}$, and for this reason the coefficients $f$ may be close to unity. This means that each molecule striking the solid surface condenses at once. The mercury halide molecules being linear have no dipole moment, and so this result is not inconsistent with Alty's empirical rule that the coefficients $f$ for non-polar molecules are near unity.

$$
\text { F. Metzger. }
$$

E. Miescher.

Physical Institute,

University of Basle. Aug. 22.

2 Alty, Proc. Roy. Soc., A, 161, 68 (1937).

Kelley, Bull., 383, U.S. Dep. of the Interior, Bureau of Mines, Washington (1935).

\section{Probable Dissociation of Nitrogen Molecules in the High Atmosphere}

KAPLAN $^{1}$ recently succeeded in exciting, in the afterglow of nitrogen, a line, $\lambda 3471 \mathrm{~A}$., that probably corresponds to the forbidden transition ${ }^{2} P \rightarrow{ }^{4} S$ of NI. Theoretically it is very interesting and curious to see that this line is emitted under relatively high pressure, at the same time as the Vegard-Kaplan bands, which are themselves forbidden.

Here, however, I wish chiefly to direct attention on the application of this beautiful experiment to the knowledge of physical conditions of the upper atmosphere. Indeed, as Kaplan points out, Dufay ${ }^{2}$ and Gauzit ${ }^{3}$ mentioned the presence of an intense radiation at a wave-length $\lambda \mathbf{3 4 7 1} \mathrm{A}$. in the spectrum of the night sky; new observations, made by me, confirm very clearly the existence of this radiation and its intensity, as it is easy to see on the accompanying spectrum. Probably we have here the line of NI, as Kaplan suggested two years ago*

The two arguments which prevented acceptance of this attribution have now disappeared. First, the

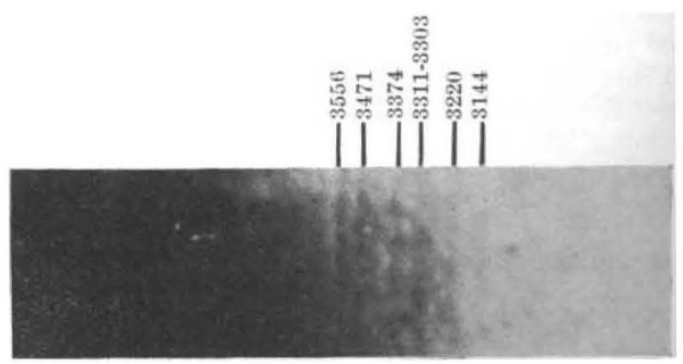

ULTRA-VIOLET SPECTRUM OF THE NIGHT SKY. 